\title{
Fundamentación teórica de la línea de investigación en educación religiosa escolar*
}

\author{
Business development and innovation in the sector of Zulian plastic manufactures \\ Fundamentação teórica da linha de pesquisa em educação escolar religiosa
}

DOI: https://doi.org/10.21803/pensam.v11i21-1.272

\author{
Vanston Irwin Archbold Hooker \\ https://orcid.org/0000-0002-4146-1378 \\ Esteban Lozano Pulido \\ https://orcid.org/0000-0002-3174-7014 \\ Hernán Berdejo González \\ https://orcid.org/0000-0001-7501-6486 \\ Javier Molina Polo \\ https://orcid.org/0000-0002-9250-397X \\ Wilfredo Mora Julio \\ https://orcid.org/0000-0003-3564-6381
}

\section{¿Cómo citar este artículo?}

Archbold, V., Lozano, E., Berdejo, H., Molina, J. \& Mora, W. (2018). Fundamentación teórica de la línea de investigación en educación religiosa escolar. Pensamiento Americano, 11(22), 147-155. DOI: https://doi.org/10.21803/pensam.v17i21-1.272

\section{Resumen}

Unfactor importanteen la formacion del ser humanosiguesiendola educacion religiosa. A pesar de las multiples estrategias sociales implementadas para opacar la importancia de la religion en la sociedad moderna y posmoderna, la religion y su impacto en la sociedad sigue en un espiral de crecimiento impresionante. Las practicas religiosas en diferentes modalidades y tendencias alrededor del mundo siguen ocupando un lugar importante en las sociedades, de alli el reconcimiento munidal de este derecho fundamental del ser humano, el derecho a la libertad religiosa. La legislacion colombiana se ha unido al clamor mundial por el reconocimiento de este derecho y se ha adherido al llamado internacional para salvaguardar este derecho fundamental a nivel nacional igualmente. No obstante, la implementacion de estarategias politicas, educativas y curriculares para defender este derecho en los colegios publicos y privados de Colombia sigue siendo un reto. Las diferentes perspectivas sobre la implementacion de estrategias pedagogicas y curriculares en las instituciones educativas del pais a nivel de la secundaria puede mejorase evitando asi mas agresiones a este derecho fundamental en Colombia. Este articulo describe la problemática de la Educacion Religiosa Escolar en el pais y hace una invitacion a que este derecho sea respetado en el sistema educativo evitando asi el monopolio de una denominacion religiosa sobre otra, o la imposicion de pensamientos seculares y humanistas que afecta el libre desarrollo de la educacion religiosa en este pais.

Palabras Claves: Educacion Religiosa, Pluralismo, Liberacion, cultura religiosa, derechos humanos.

\begin{abstract}
An important factor in the formation of the human being remains religious education. Despite the multiple social strategies implemented to overshadow the importance of religion in modern and postmodern society, religion and its impact on society remain in a spiral of impressive growth. Religious practices in different modalities and trends around the world continue to occupy an important place in societies, hence the worldwide recognition of such a fundamental right of the human being, the right to religious freedom. Colombian legislation has joined the world clamour for the recognition of this right and has adhered to the international call to safeguard this fundamental right at the national level as well. Nonetheless, the implementation of political, educational and curricular strategies to defend this right in public and private schools in Colombia remains a challenge. The different perspectives on the implementation of pedagogical and curricular strategies in the educational institutions of the country at the secondary level can be improved, thus avoiding more aggressions to this fundamental right in Colombia. This article describes the problem of School Religious Education in the country and
\end{abstract}

* Articulo original derivado de un proyecto de investigación: "Fundamentación Teórica la Línea de Investigación en Educación Religiosa Escolar," realizado por la Corporación Universitaria Adventista, con la asesoría del departamento de investigación de dicha Corporación con sede en Medellín durante los años 2016-2017. Realizado en la ciudad de Medellín entre febrero del 2015 a septiembre del año 2016. Costo total de la investigación son 2.000.000 de pesos. 
invites that this right is respected in the educational system thus avoiding the monopoly of one religious denomination over another or the imposition of secular and humanistic thoughts that affects the free development of religious education in this country.

Key words:Religious Education, Pluralism, Liberation, Religious culture, human rights.

\section{Resumo}

Um fator importante na formação do ser humano continua a ser a educação religiosa. Apesar das múltiplas estratégias sociais implementadas para opacar a importância da religião na sociedade moderna e pós-moderna, a religião e seu impacto na sociedade segue em um espiral de crescimento impressionante. As práticas religiosas em diferentes modalidades e tendências ao redor do mundo seguem ocupando um lugar importante nas sociedades. Desde aí o reconhecimento mundial deste direito fundamental do ser humano, o direito à liberdade religiosa. A legislação colombiana se uniu ao clamor mundial pelo reconhecimento deste direito e aderiu ao chamado internacional para salvaguardar este direito fundamental igualmente a nível nacional. Não obstante, a implementação de estratégias políticas, educativas e curriculares para defender esse direito nos colégios públicos e privados da Colômbia segue sendo um desafio. As diferentes perspectivas sobre a implementação de estratégias pedagógicas e curriculares nas instituições educativas do país a nível de secundária pode melhorar-se evitando assim mais agressões a este direito fundamental em Colômbia. Este artigo descreve a problemática da Educação Religiosa Escolar no país e faz um convite a que este direito seja respeitado no sistema educativo evitando assim o monopólio de uma denominação religiosa sobre a outra, ou a imposição de pensamentos seculares e humanistas que afetam o livre desenvolvimento da educação religiosa neste país.

Palavras clave: Educação Religiosa, Pluralismo, Liberação, cultura religiosa, direitos humanos.

\section{Perfil}

Vanston Irwin Archbold Hooker

Doctor en Teología del Seminario Adventista Latinoamericano (SALT) con sede en Argentina, actualmente se desempeña como Decano y director de investigación de la Corporación Universitaria Adventista, teologia@ unac.edu.co

\section{Perfil}

Estudiantes del 10 semestre de Licenciatura en Teología. Corporación Universitaria Adventista: Facultad de Teologia
Ph.D en Teología

\section{Esteban Lozano Pulido}

Hernán Berdejo González

Javier Molina Polo

Wilfredo Mora Julio 


\section{Introducción}

L

a Educacion Religiosa Escolar ha sido objeto de investigación y dimensión de práctica de diferentes teorías, intereses y perspectivas. En la bibliografía actual sobre la $E R E$, se puede encontrar que se fundamenta en la legislación o el marco jurídico que la promociona o la condiciona, la experiencia espiritual y la intencionalidad formativa que configuran las cosmovisiones de las diferentes instituciones educativas, el contexto particular y las necesidades específicas de cada región o país, la cercanía o la distancia entre la ERE y la catequesis, la diversidad religiosa que configura una problemática social, la historia de la ERE en relación con la sociedad y la política, así como diversas teorías, teologías, pedagogías, didácticas y representaciones que pretenden hacer un aporte pertinente. Para hablar de ERE resulta primordial encontrar las definiciones de esta. Meza y Castiblanco (2012) la definen así, "La ERE (Educación Religiosa Escolar), es una disciplina escolar, como tal atiende al conocimiento de la realidad religiosa y la construcción de un saber sobre la experiencia religiosa." Villareal (2013), señala:

Es una enseñanza que trabaja sobre el fenómeno religioso como experiencia humana y social, forma en el discernimiento y la compresión en función de opciones religiosas libre y maduramente. Presenta la interpretación, desde cada una de las formas religiosas, del hecho religioso y sus realizaciones, educa para la convivencia y el respeto por los valores religiosos y propone una orientación ética de la vida. Y ayuda a la construcción del hombre nuevo.

Por la misma línea Lara, Casas, Garavito, Meza y Reyes (2015) dicen que es "aquella que busca la formación en la comprensión, alcance y ejercicio de la libertad desde lo religioso como forma de concretar las dimensiones transcen- dente, espiritual, religiosa y de sentido del sujeto." López (2004) dice de la ERE:

La educación religiosa se convierte en una disciplina curricular que tiene como objetivo la humanización del estudiante, es decir contribuir a su desarrollo moral y espiritual. En esta modalidad, por ejemplo, se selecciona un objeto religioso: una estatua de la religión budista, un cuadro de la religión católica o un pasaje bíblico, se lo analiza en clase y de esta forma se despierta la curiosidad de los estudiantes para que cuestionen sus valores, profundicen en los distintos sentidos de identidad religiosa y desarrollen empatía hacia los otros. Se pretende contribuir al crecimiento moral y en valores del estudiante. Aprender desde las religiones abre la mente de los educandos y le permite comprender a los estudiantes la cultura y estilo de vida de otras personas.

Con respecto a la presentación de algunos elementos históricos de la educación religiosa en Colombia. Álvarez (2011), refleja la lucha de poderes políticos e intereses de la Iglesia Católica por el desarrollo y control de la educación en el naciente Estado. No se puede hablar de ERE en Colombia sin tener en consideración lo que menciona López (2014):

La Educación Religiosa Escolar se configuró en el marco de una disputa política por el poder, donde existía cierto interés por mantener una hegemonía política, social y cultural en la sociedad colombiana. En este contexto surge una relación de poder entre la Iglesia y el Estado en el ámbito educativo donde se dan una serie de lineamientos y orientaciones que le permiten a la Iglesia en ciertos momentos de la historia enseñar bajo una filosofía y principios netamente cristianos (p.14).

La Enseñanza Religiosa Escolar en Colom- 
bia se ha desarrollado de acuerdo con los múltiples cambios que se producen al interior de algunos estamentos sociales y políticos. La historia nacional, en los últimos años ha dejado entrever algunos elementos que denotan su importancia y trascendencia en cuanto a la relación de poder entre el Estado y la Iglesia colombiana (p.17).

\section{Objetivos}

El objetivo principal de este articulo es concientizar a la sociedad en general acerca de un derecho fundamanental de todos los seres humanos, el derecho a la libertad de conciencia, la libertad religiosa que debería permear la sociedad en general. En Colombia, ese derecho esta garantizado en la constitución permitiendo asi el libre ejercicio de las practicas religiosas. En los colegios publicos de Colombia, no obstane, el derecho puede estar siendo vulnerado por la falta de un programa de estudio para jovenes de otras denominaciones cristianas a la Iglesia Catolica. Ante el aumento galopante de una sociedad pluralista en cuanto a la religion, se hace necesario una reflexión de esta naturaleza en los circulos educativos de nuestra sociedad.

\section{La ere y la iglesia católica}

La iglesia católica ha sido preeminente en el proceso de desarrollo de la ERE en Colombia, esta define la ERE como:

Es, ante todo, un aspecto del derecho a la educación, que tiene como base una concepción antropológica abierta a la dimensión transcendente del hombre y la mujer. Unida a una formación moral, la enseñanza escolar de la religión católica favorece también el desarrollo de la responsabilidad personal y social y las demás virtudes cívicas para el bien común de la sociedad.
El Concilio Vaticano II recuerda que: «[a los padres] corresponde el derecho de determinar la forma de educación religiosa que se ha de dar a sus hijos, según sus propias convicciones religiosas. [...] Se violan, además, los derechos de los padres, si se obliga a los hijos a asistir a lecciones escolares que no corresponden a la persuasión religiosa de los padres, o si se impone un único sistema de educación del que se excluye totalmente la formación religiosa». Esta afirmación halla corroboración en la Declaración universal de derechos humanos y en otras declaraciones y convenciones de la comunidad internacional (Concilio Vaticano II, 1965).

En Colombia la Conferencia Episcopal presenta:

El estudio de la revelación cristiana y su experiencia religiosa, tomada en sus diversos componentes y manifestaciones histórico-culturales, en nuestro contexto y el de occidente. Este estudio, realizado con métodos escolares, se orienta a identificar las características de esa experiencia religiosa, sus formas de vivencia y expresión, su aporte a la humanización y al bien de la sociedad y la cultura, de manera que los alumnos elaboren sus propias conclusiones y actitudes debidamente motivados y responsables ante ese hecho religioso (Conferencia Episcopal, 1997, p.16).

\section{Marco legal}

Con la llegada la Constitución Política de 1991, López señala que la iglesia católica realiza:

El documento "Orientaciones Pastorales y Contenidos para los programas de Enseñanza Religiosa Escolar", que fue posteriormente desarrollado en dos guías tituladas "Guía para el desarrollo de los programas

Pensamiento Americano Vol. 11 (22) • 2018 • Julio-Diciembre • Corporación Universitaria Americana • Barranquilla, Colombia • ISSN: $2027-2448$. 
de Educación Religiosa en Básica Primaria" (1993) y "Guía para el desarrollo de los programas de Educación Religiosa en Básica Secundaria y Educación Media" (1993). Con base en estos programas se ha hecho la formación de los profesores, la elaboración de textos y ayudas didácticas para la enseñanza religiosa, bajo un enfoque netamente católico. La enseñanza religiosa escolar responde a las dimensiones antropológica, histórica, cultural, ética, psicológica y social, constitutivas de la persona humana y su relación con la escuela en sus dimensiones pedagógica, epistemológica y legal (Ley 115, 1994. Art. 23 y 24). Al lado de los fundamentos educativos y pastorales de la educación religiosa escolar, se debe mencionar el derecho de los alumnos a una educación básica completa. Que incluye una educación religiosa acorde con la propia confesión y con el patrimonio cultural más significativo de nuestro contexto. (Const., 1991. Art. 19; Decreto 94, 1991 y Art. 26 de la Declaración Universal de los Derechos Humanos) (López, 2014).

El aspecto de la ERE, en la persona humana es algo constitutivo; no es marginal ni circunstancial, por eso que la Ley general de Educación propone la enseñanza religiosa como área fundamental, en cuanto ofrece una formación acorde con las necesidades de la persona en su proceso de aprendizaje.

La ERE en Colombia, nos presenta unas características de tipo ideológico metodológica; así:

\section{Liberadora}

En Colombia se desarrolla la investigación en ERE desde algunas perspectivas tales como la liberadora, basada en la teología de la liberación. En este sentido algunos autores proponen un enfoque de ERE bajo una perspectiva liberadora.
Una ERE liberadora asume la finalidad de la educación religiosa como es: de una parte, "favorecer el desarrollo integral de la persona, el logro de su propia autonomía $y$ el de su identidad personal y social" y de otra, promover las dimensiones espiritual y religiosa en su relación con la cultura, la sociedad y la religión." Como parte de este cometido, se espera que el sujeto forme un pensamiento reflexivo, analítico y crítico sobre los problemas religiosos de su realidad; sepa dar sentido a la existencia última de su vida; integre fe y vida en lo cotidiano; establezca relaciones dialógicas con los otros; en últimas, viva su vocación mediante de su propia humanización y la humanización del mundo (Suárez et al., 2013).

Por la misma línea, Un proyecto financiado por la Pontificia Universidad Javeriana y dirigido por José Luis Meza y Gabriel Suárez, de la Facultad de Teología, como investigadores principales, junto con los coinvestigadores Juan Alberto Casas, Daniel de Jesús Garavito, José Orlando Reyes y David Eduardo Lara, presentan un artículo el cual recoge las intuiciones del grupo de investigación en torno a la importancia de la ERE, para la formación de la dimensión religiosa del sujeto y la lectura crítica de la realidad (Lara et al, 2015). Se deriva de la investigación sobre la ERE liberadora, que pretende identificar los elementos liberadores en la ERE presentes en algunas instituciones educativas. Presentan en un contexto de la modernidad una fundamentación desde la teología de la liberación y pedagogía liberadora, presentando su objeto de estudio como la libertad de culto, valorando las tradiciones que han configurado la cultura y la historia que otorgan identidad a las personas.

Otro autor ahonda más en detalles en la condición de las necesidades sociales y por ende en su énfasis de liberadora (Meza Rueda, 2016), plantea que la pobreza, la injusticia, la

Pensamiento Americano Vol. 11 (22) • 2018 • Julio-Diciembre • Corporación Universitaria Americana • Barranquilla, Colombia • ISSN: $2027-2448$. 
violencia y tantos otros fenómenos de nuestro continente son expresión de que la lógica comunicada por la cultura (instrumentalización del sujeto y consumismo) y la religión (pasividad, determinismo y patriarcalismo) merecen ser problematizados en la escuela y, de manera particular, por la educación religiosa. Si la liberación consiste en "una liberación social, política, económica, cultural; liberación humana en sus diferentes aspectos; liberación del pecado" entonces, hoy más que nunca adquieren vigencia las intuiciones de la teología de la liberación y la pedagogía liberadora. Por tal motivo, se quiere establecer los principios de una ERE liberadora que contribuya a la emancipación del sujeto y potencialice su dimensión religiosa dentro de una perspectiva espiritual que no puede negar la necesidad de restablecer lo inmanente (la política, la economía y la cultura).

\section{Pluralista}

Otra perspectiva en investigación de la ERE es la del pluralismo, (Coy, 2010) presenta un artículo donde hace una aproximación del nuevo paradigma para Colombia en el cual se instala el pluralismo religioso, que conlleva a buscar un cambio de mentalidad, de currículos y métodos con el fin de que las instituciones educativas se enfoquen en proveer a los estudiantes de competencias religiosas y no se enfoque en crear adeptos de una religión. Guzmán y Arias (2009), en el mismo enfoque pluralista describen que la ERE ha sido, por tradición, parte de la formación escolar en nuestras sociedades latinoamericanas. Hasta hace no más de 25 años, la formación cristiana y específicamente católica, era hegemónica en el sistema escolar colombiano.

Para la actualidad, y después de un exhaustivo diseño normativo, la educación religiosa escolarizada sigue en el debate público, ya no sólo desde el ejercicio de los derechos y las li- bertades sino además desde abordajes epistemológicos y disciplinares. En esta labor participan de modo interdisciplinar, además de las instituciones eclesiales, ponentes de la pedagogía, la jurisprudencia y otras disciplinas de las ciencias sociales (psicología, sociología). El prospecto planteado en torno al tema aboga más por una educación incluyente antes que por una imposición institucional y doctrinal; siendo así un espacio para el dialogo religioso y pluralista que acoja a toda persona en la diferencia de creencias.

Guzmán (2010), plantea en su artículo un estudio de la ERE desde la perspectiva transicional de Colombia de la modernidad a la posmodernidad donde manifiesta la necesidad de impartir una ERE pluralista y que atienda al lenguaje simbólico de la tradición religiosa y cultural colombiana. En los últimos años se ha visto un incremento de las propuestas en ERE., dado que se amplían los espacios de participación pluralista religioso en la sociedad, al tiempo que incrementa el secularismo en la población, esto abre un espacio al camino de religiones comparadas y un análisis crítico del discurso de las religiones como plantea White (2004), que pretende ampliar el espectro académico de la religión instalando el rigor académico al momento de interpretar los textos sagrados y todo lo concerniente a las religiones preponderantes de la actualidad.

Bajo el lente de diversidad de la sociedad colombiana, Moncada (2015), en su artículo plantea que en el mundo entero todos somos diferentes, en el proceso de la educación funciona la misma lógica. En los últimos tiempos, con la mundialización y con la aceleración de las migraciones, el mundo ha cambiado radicalmente, y la diversidad religiosa es visible y experimentable en todo el mundo. Las religiones están hoy presentes virtualmente ante todas las demás. Los creyentes de unas y otras se encuentran cotidianamente, en la calle, en

Pensamiento Americano Vol. 11 (22) • 2018 • Julio-Diciembre • Corporación Universitaria Americana • Barranquilla, Colombia • ISSN: $2027-2448$. 
el trabajo, en los bloques de vivienda, en los lazos de la familia extensa, en los medios de comunicación. Entonces se podría realizar la pregunta ¿para qué una ERE pluralista? Pues nos permite determinar que el objetivo pedagógico de esta área del conocimiento no radicaría en adoctrinar o catequizar a un grupo de estudiantes en una tradición de fe particular, por el contrario, estaría llamada a concienciar, a dar contenidos y a generar escenarios de reflexión que permitan a los estudiantes construir criterios claros frente a una realidad en la cual no solo se encuentran inmersos, sino que también emerge de su constitución socio- antropológica. Más a fondo, en la perspectiva pluralista también se abre campo a la diversidad cognitiva en la ERE. Bernal (2013), plantea una propuesta pedagógica que, con fundamento en la diversidad cognitiva, otorga reconocimiento al pluralismo religioso en los ambientes escolares y particularmente en la clase de Religión, desde las diferentes posturas pedagógicas que operan con una variedad metodológica.

\section{PSICOLÓGICA}

Cuéllar e Imbachi (2016), presentan en su artículo una perspectiva psicológica de la ERE donde se plantean elementos de importancia como el sentido de la vida, la trascendencia, y la resiliencia. Que busca atender un medio donde se adolece de pluralismo religioso o se presenta el fenómeno de la no existencia de creencias religiosas. En cuanto al sentido de la vida presenta la creencia religiosa no solo como medio para entender el medio que lo rodea, sino como algo que le permite dar sentido a su realidad y de cómo interactúa con ella; para la trascendencia toma en cuenta la experiencia particular y de cómo entiende lo sobrenatural y por último el aporte psicológico de la resiliencia al acercarse a la realidad del individuo con esperanza, pues al estar enmarcada en la violencia, la corrupción, y otras tan- tas patologías sociales, donde como propuesta pedagógica abre el horizonte de sentido para formar hombres y mujeres capaces de superar adversidades cotidianas, generando una nueva conciencia que permita respetar la diversidad religiosa.

\section{Histórico-cultural}

En este enfoque Bonilla Morales y García Garzón (2014), plantean un modelo históricocultural, donde refleja que el desarrollo cognitivo de los individuos esta mediado socialmente, uno de estos se da por el estímulo religioso que no se puede desligar de la construcción de la realidad integral del ser humano. Se aborda el discurso desde los relatos históricos y su contexto cultural para adaptar sus enseñanzas a la vida actual. Se encuentran dos posturas histórico-culturales, la primera es usada por las instituciones educativas oficiales del Estado, que toman el relato cristiano desde una perspectiva historica y hace una comparación cultural de valores y costumbres desde antaño hasta hoy, eso permite construir un enfoque cristiano para la ERE en Colombia.

La segunda postura histórico-cultural propone un estudio de las religiones preponderantes en el mundo para abordarlas desde una perspectiva histórica y destacando sus aportes culturales de cada época, pero dejando de lado lo confesional, enfocándose así en un estudio académico de las religiones no proselitista.

\section{Evangelizadora}

En una perspectiva evangelizadora, Moreno (2015) en su artículo enfatiza que "La iniciativa del sistema educativo de incorporar el sistema de educación religiosa, es muy valiosa dado que se aterriza el punto de evangelismo en los planteles formativos, dado que esto contribu-

Pensamiento Americano Vol. 11 (22) • 2018 • Julio-Diciembre · Corporación Universitaria Americana • Barranquilla, Colombia • ISSN: 2027-2448 • 
ye a promover y reformar las academias desde la etapa inicial. La enseñanza religiosa es un aspecto fundamental en la formación integral de la persona y un elemento imprescindible en el ejercicio del derecho de libertad religiosa y de conciencia. La religión como "creencia en Dios" es un buen ejemplo y más cuando se asume una actitud positiva para dar aportes significativos frente al proceso que se vive en nuestro país del conflicto.

La iglesia católica desde que se fundó ha tenido gran influencia en el ámbito mundial, pero a partir de la última década su objetivo es evangelizar a todas las poblaciones, sin importar su cultura, aunque la relación entre la ERE y el sistema educativo son débiles y pobre, se busca afianzar esta unión. Dando como resultado un mundo más unido, más preocupado por las necesidades del prójimo, fortaleciendo los valores y las prácticas religiosas que se han perdido".

\section{Humanista}

Otra perspectiva encontrada es la humanista, de la cual Cárdenas y Castañeda (2011) presentan una ERE donde se destaca una interpretación humanista del Nuevo Testamento que se vincula con la teología liberadora, pero que difiere de ella en su metodología. Es un proceso de investigación para construir vida, amor, sentidos, significados y pensamiento social. Es una perspectiva que busca formar seres humanos con una ética autónoma y mentalidad mundial, con responsabilidad social, amantes de sí mismos, del otro, del mundo y naturalmente amantes del conocimiento, cuyo propósito esencial es la formación de un ser humano con madurez mental.

\section{Conclusiones}

La educación religiosa en Colombia tiene por delante grandes retos, en primer lugar, debe luchar contra el control político y religioso del catolicismo que en su afán de fortalecer la fe de sus adeptos, impone programas de formación religiosa sin considerar las diferencias religiosas en las comunidades escolares, especialmente en las escuelas públicas. En segundo lugar, la educación religiosa en Colombia, como en otros países del mundo, aunque se reconoce su importancia, tiende a la secularización en donde la religión y sus valores son remplazadas por ideas netamente humanistas. Ante esta situación se requiere de un mayor esfuerzo por parte del Ministerio de Educación Nacional para salvaguardar los derechos de los jóvenes en edad escolar a una educación religiosa que respete sus diferencias y tendencias religiosas, libre de imposiciones y presiones tanto religiosos como políticas. 


\section{Referencias}

Álvarez, M. (2011). Religión, política y educación en Colombia. La presencia religiosa extranjera en la consolidación del régimen conservador durante la Regeneración. Revista de Historia Regional y Local 3(6). 154-172.

Bernal, N. (2013). Pedagogía De La Diversidad Cognitiva: Una Propuesta Para El Reconocimiento Del Pluralismo En La Educación Religiosa Escolar. Tesis de Especialización en Educación Religiosa Escolar. Bogotá, Colombia: Universidad de San Buenaventura.

Bonilla Morales, J. \& García Garzón, J. (2014). Educación Religiosa Escolar y Pedagogías para el Reconocimiento del Pluralismo Religioso. Bogotá, Colombia: Universidad de San Buenaventura.

Cárdenas, A. \& Castañeda, J. (2011). El Maestro de ERE Desde la Mirada De Jesús Buen Pastor Y de la Pedagogía de la Humanización. Tesis de Especialización en Educación Religiosa Escolar. Bogotá, Colombia: Universidad de San Buenaventura.

Concilio Vaticano II (7 de diciembre de 1965). Declaración sobre la libertad religiosa Dignitatis humanae, n. 5; cfr. Código de Derecho Canónico, can. 799; SANTA SEDE, Carta de los derechos de la familia (24 de noviembre de 1983), art. 5, c-d.

Conferencia Episcopal de Colombia. (1997). Guía para el desarrollo de los contenidos de la enseñanza religiosa escolar en los niveles de basica secundaria y media. Bogotá: Colombia: Editorial Kimpres Ltda.

Congreso de Colombia. (8 de febrero de 1994). Ley general de educación. [Ley 115 de 1994]. DO: 41.214

Coy, E. (2010). La educación religiosa escolar en un contexto plural Reflexiones preliminares, Franciscanum Revista de las ciencias del espíritu, 52(154). 53-83.

Cuéllar, N. \& Imbachi, C. (2016) Sentido de la vida y trascendencia humana, aportes al fundamento epistemológico de la ERE desde la psicología de la religión. Actualidades Pedagógicas, (68). 179-197. http:// dx.doi.org/10.19052/ap.3825

Guzmán, A. (2010). ¿Auspiciar o Controvertir la Libertad Religiosa Desde la Educación? Aportes al Debate Sobre una Educación Religiosa Pluralista. Theologica Xaveriana 60(170). 395-415.

Guzmán, S. \& Arias, Y. (2009). La educación religiosa esco- larizada. Revista Páginas, 84. 33-48.

Lara, D., Casas, J., Garavito, D., Meza, J. \& Reyes, O. (2015). Educación religiosa escolar, una mediación crítica para comprender la realidad. Magis 7.15

López, J. (2014). La educación religiosa escolar en Colombia: su enseñanza en un contexto pluralista y humanizante. Tesis de Maestría en Educación. Medellín, Colombia: Universidad de Antioquia.

Meza Rueda, J., Suárez, G., Casas, J., Garavito, D., Lara, D. \& Reyes, J. (2016). ¿Religión en la escuela? Si es liberadora, sí: repensar la educación religiosa a partir de la teología y la pedagogía de la liberación. Revista Internacional de Educación Preescolar e Infantil 1(1). $1-18$.

Meza, J. L. \& Castiblanco, J. (2012). Educación Religiosa Escolar: Naturaleza, Fundamentos y Perspectivas. Bogotá, Colombia: San Pablo.

Moncada, C. (2015). Colombia diversa, tarea para la ERE. Memorias I Coloquio de Educación Religiosa Escolar: Tensiones Tendencias y Perspectivas, Colombia, octubre 30 y $31.31-37$.

Moreno, O. (2015). Aportes de la educación y de la educación religiosa escolar a la evangelización ya la cultura en Colombia 2000-2013. Memorias I Coloquio de Educación Religiosa Escolar: Tensiones Tendencias y Perspectivas. Colombia, octubre 30 y 31 de 2015.

Presidencia de Colombia. (20 de enero de 1992). Convención sobre los Derechos del Niño y la Reserva formulada por Colombia respecto de su artículo 38, numerales, $2^{\circ}$ y $3^{\circ}$. [Decreto 94 de 1992]. DO: 40.290

Suárez, G., Meza, J., Garavito, D., Lara, D., Casas, J. \& Reyes, J. (2013). Educación Religiosa Escolar en Clave Liberadora: Elementos Constitutivos. Theologica Xaveriana 63(175). 219-248.

Villareal, N. (2013). La Liturgia Eucarística Como Pedagogía de la Fe. Tesis de Grado en Licenciatura en Teología. Bogotá, Colombia: Pontificia Universidad Javeriana.

White, J. (2004). Should religious education be a compulsory school subject? British Journal of Religious Education 26(2) 151-164.

2018, Vol. 11(22) 147-155. (CThe Author(s) 2018 Reprints and permission: www.americana.edu.co

https://www.coruniamericana.edu.co/publicaciones/ojs/index.php/pensamientoamericano/index 